\title{
RELACIÓN ENTRE LA FERTILIZACIÓN FOLIAR Y EL RENDIMIENTO DEL FRUTO DE OCRA (Abelmoschus esculentus) 1
}

\author{
Arturo Díaz Franco ${ }^{2}$, Alfredo S. Ortegón M.2
}

\begin{abstract}
RESUMEN
Relación entre la fertilización foliar y el rendimiento del fruto de ocra (Abelmoschus esculentus). Con el propósito de conocer el impacto de la aplicación de fertilizantes foliares comerciales sobre el rendimiento de fruto de ocra (Abelmoschus esculentus), variedad 'Clemson Spineless 80', se realizaron estudios de campo en 1994 y 1995. En 1994 se evaluaron Carboxy y Foltron al follaje y Profit-G aplicado al suelo, así como sus combinaciones en suelo fertilizado. En 1995, los fertilizantes Carboxy, Foltron y Mora-Leaf se aplicaron al follaje en dosis altas y en suelo sin fertilizar. En todos los casos, se realizaron cinco aspersiones a intervalos de siete a 10 días iniciadas durante la primera semana de cosecha. En 1994, los tratamientos no mostraron efectos significativos sobre el peso de fruto, el rendimiento comercial de fruto y la altura de planta, comparado con el testigo. En 1995 no se observó influencia en la altura de planta y en el rendimiento de fruto. No hubo efecto de la fertilización foliar en el rendimiento de fruto en ocra.
\end{abstract}

\section{ABSTRACT}

Relationship between foliar fertilization and okra (Abelmoschus esculentus) fruit yield. Field studies were carried out during 1994 and 1995, in order to determine the impact of applying commercial foliar fertilizers on the 'Clemson Spineless 80" okra fruit variety (Abelmoschus esculentus). Carboxy and Foltron applied on folliage, and Profit-G applied on the soil, as well as their combinations applied on fertilized soil were evaluated in 1994. Carboxy, Foltron and Mora-Leaf fertilizers were applied to folliage in high dosages and on non-fertilized soil during 1995. All plants were sprayed five times in 7 to 10-day intervals starting the first week of harvest. When compared to control plants, treatments applied during 1994 did not have a significant effect on fruit weight, fruit commercial yield and plant height. Treatments applied during 1995 did not influence plant height or fruit yield. The study proved that foliar fertilization does not have an impact on okra fruit yield.

\section{INTRODUCCIÓN}

En el valle del norte de Tamaulipas el cultivo de ocra (Abelmoschus esculentus L.) Moench), conocida también como bombó, bumbó, quimbombó, etc., es la hortaliza que se siembra tradicionalmente. La ocra posee la mayor superficie cultivada en México, además es de importancia socioeconómica debido a la mano de obra generada y las divisas por la exportación del fruto. Los suelos de la región son, en general, carentes de $\mathrm{N}$ y P (Loera y Martínez, 1987), por lo que el productor de ocra comúnmente adiciona a sus suelos dichos elementos. Sin embargo, la fertilización foliar es una práctica generalizada que realiza el productor en repetidas ocasiones, con o sin mezclas de pesticidas, al asumir que incrementa el rendimiento de fruto. Existe en el mercado gran número de fertilizantes foliares que contienen diversos elementos y cuyos fabricantes aseguran beneficios en la productividad. La fertilización foliar representa un método atractivo ya que, en teoría, se elimina la ineficiente obtención de los nutrimentos por la raíz y éstos por el contrario, se mueven directamente dentro de la planta a través de las hojas (Wiedenfeld, 1988).

La información sobre la nutrición mineral de la ocra es limitada principalmente porque es una hortaliza "menor" y para obtener datos experimentales, requiere largos periodos de cosecha y gran cantidad de mano de obra. La aplicación foliar de zinc en ocra resultó efectiva sólo en suelos con alto contenido de P (Hipp y Cowley, 1971). La respuesta a los fertilizantes foliares en otros cultivos ha sido variable. La aplicación de

1 Forma parte del proyecto de investigación $\mathrm{N}^{\circ} 323$ con el apoyo del Patronato para la investigación, fomento y sanidad vegetal.

2 Campo Experimental Río Bravo, INIFAP. Apdo. Postal. 172, Río Bravo, Tam., 88900, México.

E-mail: diaza@cirne.inifap.conacyt.mx 
Carboxy y Profit-G en tomate (Lycopersicon esculentum Mill.), en melón (Cucumis melo L.) y en pepino (Cucumis sativus L.) incrementaron el rendimiento (Rangel, 1993; Roman y Gutiérrez, 1993). Bayfolan Plus incrementó el rendimiento y la calidad del fruto en manzano (Malus sylvestris Mill.) (Amado y Domínguez, 1987). Biozyme incrementó el rendimiento en chile (Capsicum annuum L.) (Belakbir et al., 1998; Csizinszky, 1990). La aplicación combinada de ProfitG al suelo más Carboxy al follaje incrementó el rendimiento de pepino, comparado al efecto independiente de dichos productos (Rangel, 1993). Por el contrario, no se observó influencia en la producción con las aspersiones de Carboxy y Foltron en brócoli (Brassica oleracea L.) (Díaz y Ortegón, 1995); de Biozyme, Biogen, Cosmofer y Cosmocel en chícharo de vaca (Vigna unguiculata L. Walp) (Díaz y Morales, 1994); así como de Bayfolan Plus en melón, sandía [Citrullus lanatus (Thunb.) Matsum. \& Nakai], y calabacita [Cucurbia роро var. melopepo (L.) Alef.] (Wiedenfeld, 1984). Por lo que el propósito del presente estudio fue el de conocer la influencia de la aplicación de diferentes fertilizantes comerciales, aplicados a partir de la primera semana de cosecha, sobre el rendimiento de fruto de ocra.

\section{MATERIALES Y MÉTODOS}

Se realizaron estudios de campo en el municipio de Río Bravo, Tamaulipas y en todos los casos se utilizó la variedad 'Clemson Spineless 80', de uso generalizado en la región. Las dosis de los fertilizantes considerados en el presente trabajo fueron según los rangos indicados por los fabricantes.

Estudios en 1994: Se establecieron dos experimentos, uno en el Campo Experimental Río Bravo, INIFAP (CERIB) y el otro en la localidad "La Posta" dentro de una siembra comercial. El análisis del suelo en presiembra, efectuado en el laboratorio de suelos del CERIB, indicó que ambas localidades el suelo fue carente de $\mathrm{N}$, P, alto en $\mathrm{K}$, bajo contenido de materia orgánica, sin problemas de salinidad y ligeramente alcalino (Cuadro 1). En la localidad CERIB se sembró el 23 de Marzo de 1994, en un suelo arcilloso, previamente fertilizado con $\mathrm{N}$ a razón de $120 \mathrm{~kg} / \mathrm{ha}$ y con $\mathrm{P}$ a $60 \mathrm{~kg} / \mathrm{ha}$, según lo señalado por Díaz (1992). Los productos evaluados fueron Profit-G aplicado en el suelo al momento de la siembra y Foltron y Carboxy aplicados al follaje (ver composición química en el Cuadro 2); el Foltron tiene uso común en la producción comercial local. Los tratamientos se agruparon de la siguiente forma: 1) Foltron, 6 1/ha; 2) Carboxy, 8 1/ha; 3) Profit-G al suelo a dosis de $10 \mathrm{~kg} / \mathrm{ha}$; 4) Profit-G (10 kg/ha) + Foltron (3 1/ha); 5) Profit-G (10 kg/ha) + Carboxy (4 1/ha); 6) Profit-G (10 kg/ha) + Foltron (3 l/ha) + Carboxy (4 1/ha); y 7) testigo, sin aplicación. En la localidad "La Posta" se sembró el 20 de Febrero de 1994, en un suelo migajón arcilloso, fertilizado con N, $90 \mathrm{~kg} / \mathrm{ha}$; $\mathrm{P}, 85 \mathrm{~kg} / \mathrm{ha}$; y K, $25 \mathrm{~kg} / \mathrm{ha}$ (a criterio del productor). En este caso, los tratamientos fueron: Carboxy a dosis de tres y seis 1/ha; Foltron a tres y cuatro 1/ha; y el testigo.

En ambos experimentos los tratamientos se distribuyeron en bloques al azar con cuatro repeticiones y las parcelas fueron de tres surcos de $0,8 \mathrm{~m}$ y seis $\mathrm{m}$ de longitud, con una densidad aproximada de 45000 plantas/ha. Los riegos se aplicaron según las necesidades del cultivo, y contra el pulgón (Aphis gossypii Glover) se aplicó malathion (1 1/ha) en plántula. Se realizaron cinco aspersiones de los fertilizantes foliares con bomba de mochila, en periodos de siete a 10 días, iniciados el 27 de mayo en la localidad CERIB y el 26 de Abril en "La Posta", periodos correspondientes a la primera semana de cosecha. El rendimiento de fruto se obtuvo de los surcos centrales, la cosecha manual (cortes) se realizó tres veces por semana. En la localidad CERIB el periodo de cosecha fue de 14 semanas y en "La Posta" fue de 11 semanas. El fruto comercial, no mayor de $12,7 \mathrm{~cm}$ de longitud, se seleccionó y los frutos mayores o "cuernos" se eliminaron. Además, se estimó el peso de fruto y la altura de planta en madurez y de cinco plantas por parcela. Los datos se analizaron estadísticamente mediante el paquete SAS (SAS Inst., Cary, N. C.).

Estudios en 1995: Se estableció un experimento el 14 de marzo de 1995 en un terreno arcilloso del Campo Experimental Río Bravo (CERIB), pobre en $\mathrm{N}$ y $\mathrm{P}$, alto en $\mathrm{K}$, bajo en el porcentaje de materia orgánica, sin problemas de salinidad y $\mathrm{pH}$ ligeramente alcalino (Cuadro 1), el cual no se fertilizó. En este estudio se evaluaron dosis altas de los fertilizantes foliares Foltron, cuatro 1/ha; Carboxy, ocho 1/ha; y Mora-Leaf, seis 1/ha (ver composición química en el Cuadro 2). Las parcelas fueron de cuatro surcos de $0,8 \mathrm{~m}$ y cinco $\mathrm{m}$ de

Cuadro 1. Resultados de los análisis de suelo en presiembra de las localidades en el estudio.

\begin{tabular}{lrcr}
\hline Análisis & \multicolumn{2}{c}{$\mathbf{1 9 9 4}$} & 1995 \\
\cline { 2 - 3 } & CERIB & La Posta & CERIB \\
\hline N (ppm) & 7,8 & 9,0 & 7,4 \\
P (ppm) & 11,7 & 10,0 & 10,5 \\
K (ppm) & 500,0 & 510,0 & 488,0 \\
MO (\%) & 1,6 & 1,0 & 1,5 \\
Salinidad & 0,9 & 1,7 & 1,0 \\
pH & 7,6 & 7,8 & 7,5 \\
\hline
\end{tabular}

* Milimohos $/ \mathrm{cm} / 25^{\circ} \mathrm{C}$. 
Cuadro 2. Composición química (\%) de los fertilizantes evaluados en ocra, según sus fabricantes.

\begin{tabular}{|c|c|c|c|c|}
\hline Nutrimento & Carboxyx & Profit-Gx & Foltrony & Mora-Leafz \\
\hline $\mathrm{N}($ total $)$ & & 4,5 & 10,0 & 20,0 \\
\hline Fósforo & & & 20,0 & 20,0 \\
\hline Potasio & & & 5,0 & 20,0 \\
\hline Fierro & & & 0,005 & 0,15 \\
\hline Magnesio & & & 0,0021 & 0,05 \\
\hline Manganeso & & & 0,014 & 0,05 \\
\hline Zinc & & & 0,0021 & 0,15 \\
\hline Cobre & & & 0,013 & 0,05 \\
\hline Azufre & & & & 0,20 \\
\hline Boro & & & & 0,02 \\
\hline Calcio & & & & 0,05 \\
\hline Sales polihidroxicarboxiladas de amonio & 5,0 & 12,5 & & \\
\hline Carbohidratos & 5,0 & 12,5 & & \\
\hline
\end{tabular}

longitud y se utilizó un diseño de bloques al azar con cinco repeticiones. La densidad de población se ajustó a 45000 plantas/ha. El número y la frecuencia de las aspersiones de los fertilizantes foliares fueron semejantes que en los estudios de 1994 y la primera aplicación se realizó el 7 de Junio, durante la primera semana de cosecha. La cosecha se efectuó también tres veces por semana y en un periodo total de siete semanas. El fruto comercial se cuantificó de los dos surcos centrales de cada parcela de donde se obtuvo el rendimiento. En madurez fisiológica se midió la altura de cinco plantas por parcela. Para determinar la significación entre los tratamientos, los datos se sometieron a análisis de varianza mediante el paquete SAS (SAS Inst., Cary, N. C.).

\section{RESULTADOS Y DISCUSIÓN}

Los resultados de los estudios en las localidades CERIB y "La Posta" en 1994 y en el CERIB en 1995, esta última sin fertilización al suelo, demostraron que los tratamientos aplicados de fertilizantes foliares, al suelo y combinados, no tuvieron influencia estadística significativa en el rendimiento de fruto comercial en la variedad 'Clemson Spineless 80'. Igualmente, no se observó efecto significativo en la altura de planta y el peso de fruto en 1994 y en altura de planta en 1995. Resultó evidente el bajo rendimiento en 1995 (3,9 t/ha) comparado con el obtenido en los experimentos del CERIB (8,6 t/ha) y "La Posta" (8,0 t/ha) en 1994 (Cuadros 3 y 4), lo cual se atribuye, entre otros factores, a la falta de fertilización en el sitio de 1995. Por el contrario, en algunos estudios se ha demostrado que el peso y la altu- ra de la planta de chile, frijol (Phaseolus vulgaris L.) y maíz (Zea mays L.) se incrementaron con las aplicaciones de fertilizantes foliares (Campos et al., 1994; Elsayed, 1995); en particular con el Profit-G y Carboxy, se incrementaron los rendimientos en el pepino, en tomate y en melón (Rangel, 1993; Roman y Gutiérrez, 1993).

Diversos factores climáticos, fisicoquímicos de la solución aplicada y metabólicos en la planta, interaccionan y pueden afectar la absorción foliar del fertilizante (Rodríguez, 1992) Las hojas no están adaptadas para absorber gran cantidad de nutrimentos, en particular N, P y K, por lo que la fertilización foliar tiende a mostrar resultados inconsistentes. Se determinó que los productos evaluados en el presente estudio, no aportaron incrementos en el rendimiento de fruto de ocra, por lo que los consideramos como "tiros de escopeta" ineficaces y por el contrario incrementan los costos de producción (Shuler y Hochmuth,1990).

La aplicación de fertilizantes foliares se justificaría sólo cuando alguna deficiencia nutrimental haya sido adecuadamente diagnosticada en el cultivo (Hochmuth y Hanlon, 1989; Rodríguez, 1992). Sin embargo, es conveniente a futuro determinar la posible influencia de la fertilización foliar y reguladores del crecimiento sobre la calidad del fruto de ocra.

\section{AGRADECIMIENTOS}

Se agradece el apoyo del Patronato para la Investigación, Fomento y Sanidad Vegetal a través del 
Cuadro 3. Respuesta de la aplicación de fertilizantes foliares y al suelo en ocra variedad 'Clemson Spineless 80’ en 1994.

\begin{tabular}{|c|c|c|c|c|}
\hline Tratamiento & Dosis (ha) & $\begin{array}{l}\text { Altura de planta } \\
(\mathrm{cm})\end{array}$ & $\begin{array}{l}\text { Peso de fruto } \\
\text { (g) }\end{array}$ & $\begin{array}{c}\text { Rendimiento } \\
\text { comercial (kg/ha) }\end{array}$ \\
\hline \multicolumn{5}{|c|}{ Localidad CERIB } \\
\hline Foltron & 61 & 137 & 10,9 & 8.613 \\
\hline Carboxy & 81 & 136 & 10,4 & 8.579 \\
\hline Profit-G & $10 \mathrm{~kg}$ & 137 & 10,7 & 8.596 \\
\hline Profit-G + Foltron & $10 \mathrm{~kg}+31$ & 140 & 10,4 & 8.500 \\
\hline \multicolumn{5}{|c|}{ Profit-G + Carboxy } \\
\hline $10 \mathrm{~kg}+41$ & 141 & 10,3 & 8.787 & \\
\hline Profit-G + Foltron & $10 \mathrm{~kg}+31+41$ & 139 & 10,5 & 8.369 \\
\hline Testigo & - & 137 & 10,3 & 8.936 \\
\hline Significación & & NS & NS & NS \\
\hline \multicolumn{5}{|c|}{ Localidad "La Posta" } \\
\hline Carboxy & 31 & 110 & 11,2 & 8.027 \\
\hline \multirow{3}{*}{ Foltron } & 61 & 106 & 12,6 & 8.103 \\
\hline & 31 & 109 & 12,1 & 7.945 \\
\hline & 41 & 108 & 11,5 & 8.075 \\
\hline Testigo & - & 114 & 11,7 & 8.166 \\
\hline Significación & & NS & NS & NS \\
\hline
\end{tabular}

NS No-significación en el ámbito de p $\leq 0,05$.

Cuadro 4. Respuesta de la aplicación de dosis altas de fertilizantes foliares comerciales en ocra ('Clemson Spineless 80'), en un suelo sin fertilizar durante 1995 .

\begin{tabular}{lccc}
\hline Fertilizante & $\begin{array}{c}\text { Dosis } \\
\text { (ha) }\end{array}$ & $\begin{array}{c}\text { Altura de } \\
\text { planta }(\mathbf{c m})\end{array}$ & $\begin{array}{c}\text { Rendimiento } \\
\text { comercial (kg/ha) }\end{array}$ \\
\hline Foltron & 41 & 107 & 3.861 \\
Carboxy & 81 & 101 & 3.897 \\
Mora-Leaf & 61 & 103 & 3.970 \\
Testigo & - & 105 & 4.203 \\
Significación & & NS & NS \\
\hline
\end{tabular}

NS No-significación en el ámbito de p $\leq 0,05$.

proyecto No. 323. También a Andrés Ruiz Acosta y Juan Olvera Martínez por su asistencia en los trabajos de campo.

\section{LITERATURA CITADA}

AMADO, A. J.; DOMÍNGUEZ,E. D. 1987. Fertilización foliar y la producción de manzano. Informe anual. Campo Experimental Sierra de Chihuahua, INIFAP. México. pp: 25-39.

BELAKBIR, A.; RUIZ,J.M.; ROMERO,L. 1998. Yield and fruit quantity of pepper (Capsicum annuum L.) in response to bioregulators. HortScience 33 (1): 85-87.
CAMPOS, C. A.; SCHEURING,D.C.; MILLER,J.C. 1994. The effect of biozyme on emergence of bean and sweet corn seedlings under suboptimal field conditions. HortScience 29: 734

CSIZINSZKY, A. A. 1990. Response of two bell pepper (Capsicum annuum L.) cultivars to foliar and soil applied biostimulants. Soil and Crop Sci. Soc. Fla. Proc. 49: 199-203.

DÍAZ, F.A. 1992. Guía para la producción de ocra. Campo Experimental Río Bravo, INIFAP. Desplegable No. 15. México.

DÍAZ, F.A.; MORALES,J. H. 1994. Efficacy of foliar fertilization on cowpea in northern Tamaulipas. Subtropical Plant Sci. 46: 29-31.

DÍAZ, F.A.; ORTEGÓN, A. M. 1995. Producción de brócoli y su relación con fertilización foliar y fecha de siembra. Biotam 7(1): 17-20.

ELSAYED, S. F. 1995. Response of 3 sweet pepper cultivars to biozyme under heated plastic house conditions. Scientia Hort. 61: 285-290.

HOCHMUTH, G.; HANLON, E.A. 1989. Commercial vegetable crop nutrient requirements. Florida Coop. Ext. Serv./Inst. Food \& Agric. Sci. University of Florida. Circular 806.

HIPP, W. B.; COWLEY, W.R. 1971. Importance of the phosphorus-zinc interactions in okra production. HortScience 6: $211-212$. 
LOERA, G.D.; MARTÍNEZ,E. M. 1987. Suelos. In: Manejo de Tierras de Riego. SARH Tamaulipas Norte/Patronato para la Investigación, Fomento y Sanidad Vegetal. Manual No. 5. México. pp. 13-17.

RANGEL, R.L. 1993. Efecto del carboxy en el rendimiento y calidad de tomate y pepino.Memorias V Congreso Nacional de Horticultura. Veracruz, Ver., México. p. 58.

RODRÍGUEZ, S.F. 1992. Fertilizantes. AGT. México. 157 p.

ROMAN, M.L.; GUTIÉRREZ,M. C. 1993. Profit-G y Carboxy para incrementar la calidad, la cantidad y la vida de anaquel en melón. Memorias V Congreso Nacional de Horticultura. Veracruz, Ver., México. p. 23.
SHULER, K. D.; HOCHMUTH, G.J. 1990. Fertilization guide for vegetables grown in fullbed mulch culture. Florida Coop. Ext. Serv./Inst. Food \& Agric. Sci. University of Florida. Circular 854.

WIEDENFELD, R. P. 1984. Foliar fertilization of cucurbits in South Texas. Jour. Rio Grande Valley Hort. Soc.37: 27-32.

WIEDENFELD, R. P. 1988. Vegetable crop fertilization. Jour. Rio Grande Valley Hort. Soc.41: 23-27. 